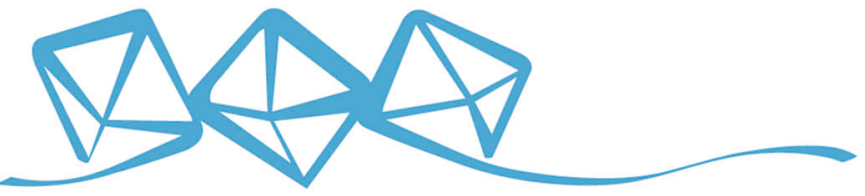 COMMUNICATIONS MATERIALS
}

ARTICLE

https://doi.org/10.1038/s43246-020-0025-2

OPEN

\section{High-cycle fatigue damage accumulation in paper}

\author{
Yoon Joo $\mathrm{Na}^{1,2}$, Sarah A. Paluskiewicz (1) ${ }^{1,2}$ \& Christopher L. Muhlstein (1) ${ }^{1,2}$
}

The mechanical durability of paper is a key consideration for applications ranging from shipping boxes to disposable medical substrates. Paper commonly experiences fatigue loading in such applications, but a high-cycle fatigue mechanism has not been identified. This research details paper's high-cycle fatigue degradation mechanism. Paper specimens were loaded with monotonically increasing, constant, and sinusoidally varying cyclic stresses, and the resulting tensile, creep, and fatigue damage accumulation rates were compared. The difficulty in defining the size and growth of cracks in paper's cellulosic fiber network were overcome with optically measured strain fields. We found that fatigue damage can accumulate via a fiber fracture mechanism, while ratchetting, creep, and tensile overload damage accumulation occurs due to failure of inter-fiber bonds. We also discovered the synergistic interaction between creep and high-cycle fatigue damage accumulation mechanisms, which is critical for extending the high-cycle fatigue life of paper.

\footnotetext{
${ }^{1}$ School of Materials Science and Engineering, Georgia Institute of Technology, Atlanta, GA, USA. ${ }^{2}$ Renewable Bioproducts Institute, Georgia Institute of Technology, Atlanta, GA, USA. ${ }^{凶}$ email: christopher.muhlstein@mse.gatech.edu
} 
$\mathrm{P}$ aper is an attractive material for a wide range of applications because it is manufactured from a renewable resource (cellulose-based wood fibers), can be readily recycled, and is biodegradable and surprisingly strong. The mechanical properties of paper are very complex due to the porous, heterogeneous network network of fibers that is predominantly hydrogen bonded $^{1,2}$. As a result, the mechanical properties of machine made paper can be anisotropic and sensitive to fiber characteristics, moisture content, temperature, and loading rate ${ }^{1}$. The subset of papers used in packaging and shipping must be mechanically robust: their mechanical response must be predictable when they are exposed to warm, moist, and mechanically-stressful conditions.

Commercial paper-manufacturing processes for packaging and print-media materials create stochastic, heterogeneous distributions of pulp fibers that bias fiber alignment toward the direction that the paper web travels through the mill. As the web moves through the mill along this "machine direction (MD)," water is removed and the fibers bond to each other. The perpendicular direction to MD is called the cross direction (CD). Commercial copy paper has a strongly bonded fiber network, so its stress-strain (constitutive) behavior is qualitatively similar to structural metals-linear, elastic (i.e., reversible) deformation is followed by permanent (plastic), nonlinear deformation that ends with catastrophic failure (Fig. 1). As one might notice by the orientation-dependent ease in tearing gift-wrapping paper, the properties of copy paper are different in the two orthogonal directions (MD and $\mathrm{CD}$ ). In fact, it is more resistant to folding and tearing perpendicular to the MD than $\mathrm{CD}$. This mechanical anisotropy comes from preferential fiber alignment in the MD: the elastic modulus, yield strength, and ultimate strength are typically larger in the MD as compared with $\mathrm{CD}$ (two to three times larger for a copy paper as we reported in ref. ${ }^{3}$ and shown in Supplementary Table 1 and Fig. 1). As the widths of copy paper specimens increased from 1.5 to $3.0,6$, and $12.5 \mathrm{~mm}$, their average (four specimens per condition) elastic moduli increased by $14 \%, 9 \%$, and $1.5 \%$, respectively. Consequently, the width dependence of the elastic modulus and yielding behavior was stable for specimens $6.0 \mathrm{~mm}$ and wider.

Tensile overload and time-dependent creep degradation in paper have been studied extensively (see ref. ${ }^{4}$ for a comprehensive review). Similarly, the short lives ( $<<100$ cycles to failure) observed during low-cycle fatigue, ratchetting, and folding have been evaluated ${ }^{5-8}$. Different modes of loading, such as tension and bending (folding), are often associated with different deformation, degradation, and failure mechanisms. Because shipping boxes can be subjected to thousands of cycles of stress as they shake during transit and delivery, we must also understand how high-cycle fatigue damage accumulates in paper. However, previous research has not fully assessed these types of conditions.

Here, we detail a tensile high-cycle fatigue damage accumulation mechanism that can degrade the durability and performance of paper-based packaging and shipping boxes that are repeatedly stressed, and we show that the mechanism is unique to cases where failures only occur after many thousands of cycles. In addition, we show that fatigue crack growth rates reveal how time-dependent and cycle-dependent mechanisms can synergistically interact to extend total life.

\section{Results and discussion}

Fracture surface evidence of damage mechanism. The mechanisms that control the deformation, degradation, and failure of wood fiber pulp-based paper and packaging materials depend on how the stresses are applied (Fig. 2). When these papers are pulled to failure in tension, the strongly bonded fiber

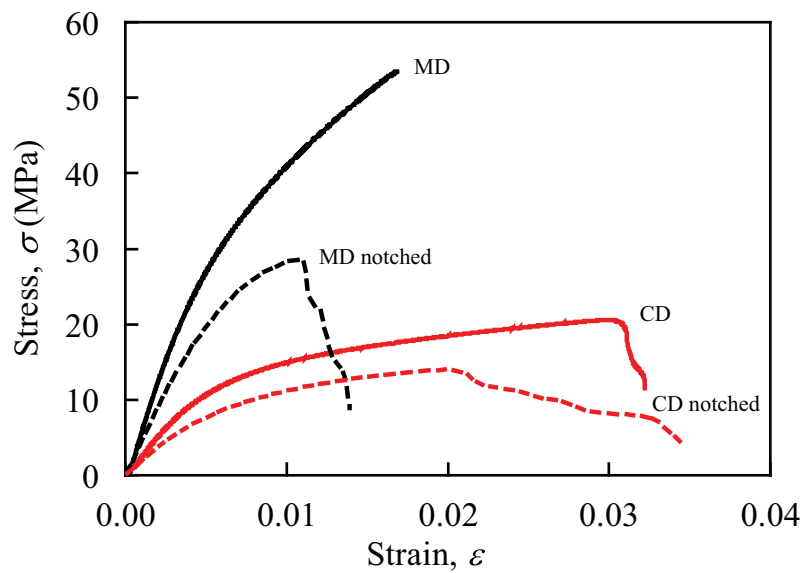

Fig. 1 Uniaxial stress-strain behavior of copy paper. The constitutive behavior was measured in the machine direction (MD) and cross direction (CD). Representative data for plain and notched $(a / w=0.27)$ tensile specimens are shown. The addition of a notch reduced the stiffness and strength of the paper (dashed lines). The error bars were within the line width.
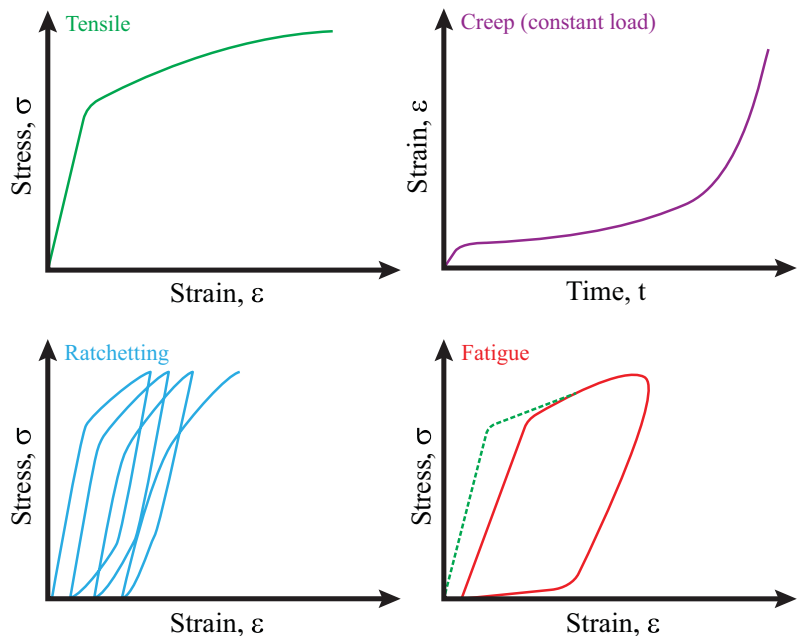

Fig. 2 Schematic description of loading modes. The amount of strain $(\varepsilon)$ that develops in a uniaxially loaded material depends on both the applied stress $(\sigma)$ and the loading conditions (tensile, creep, ratchetting, and fatigue).

network deforms (first elastically, then plastically), and fibers move relative to one another. This behavior cannot continue indefinitely-eventually the interfiber bonds fail and the paper breaks. The resulting fracture surfaces are typically hairy (Figs. 3a and 4), providing clear evidence of interfiber bond failure and the fiber pull-out fracture mechanism during tensile failure. Similarly, the fracture surfaces of folded (or creased) papers that are then torn show some fiber damage (typically crushing and buckling), but are also hairy because they fail by a similar tensile failure mechanism. A constant load can cause time-dependent damage via processes known as creep, again causing fracture surfaces that are hairy in appearance (Fig. 3e) because of a time-dependent interfiber bond failure and fiber pull-out fracture mechanism. We also know that repeated loading and unloading of paper can create cycle-dependent (as opposed to time-dependent) damage, but in this case it has not been clear if the mechanisms are distinct from the ones that control conventional tensile and creep fractures. 


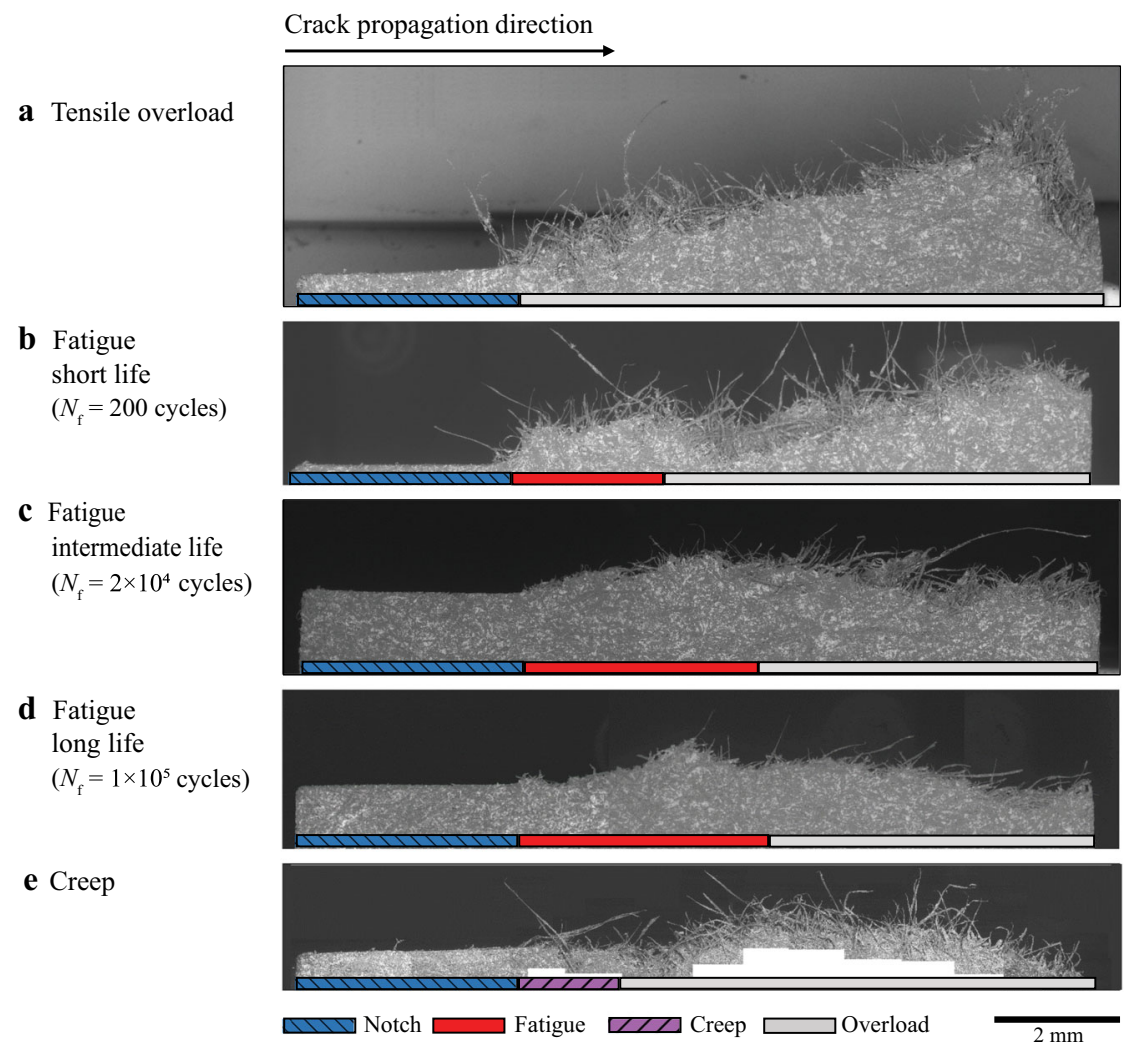

Fig. 3 Tensile overload, fatigue, and creep crack profiles. Scanning electron microscopy images of representative crack profiles the of notched paper specimens that failed during $\mathbf{a}$ tensile, $\mathbf{b}, \mathbf{c}$, and $\mathbf{d}$ fatigue, and $\mathbf{e}$ creep tests. The cracks grew completely across the specimen from the cut notch tip as indicated in the figure. Tensile overload and creep fractures are fibrous (hairy) because the interfiber bonds fail and fibers pull out. In contrast, fatigue damage causes fiber fracture and less fibrous crack profiles. The various parts of the crack path (notch, fatigue, creep, and overload) for each specimen were determined using digital image correlation and can be identified using the horizontal bar under each crack profile and the legend.

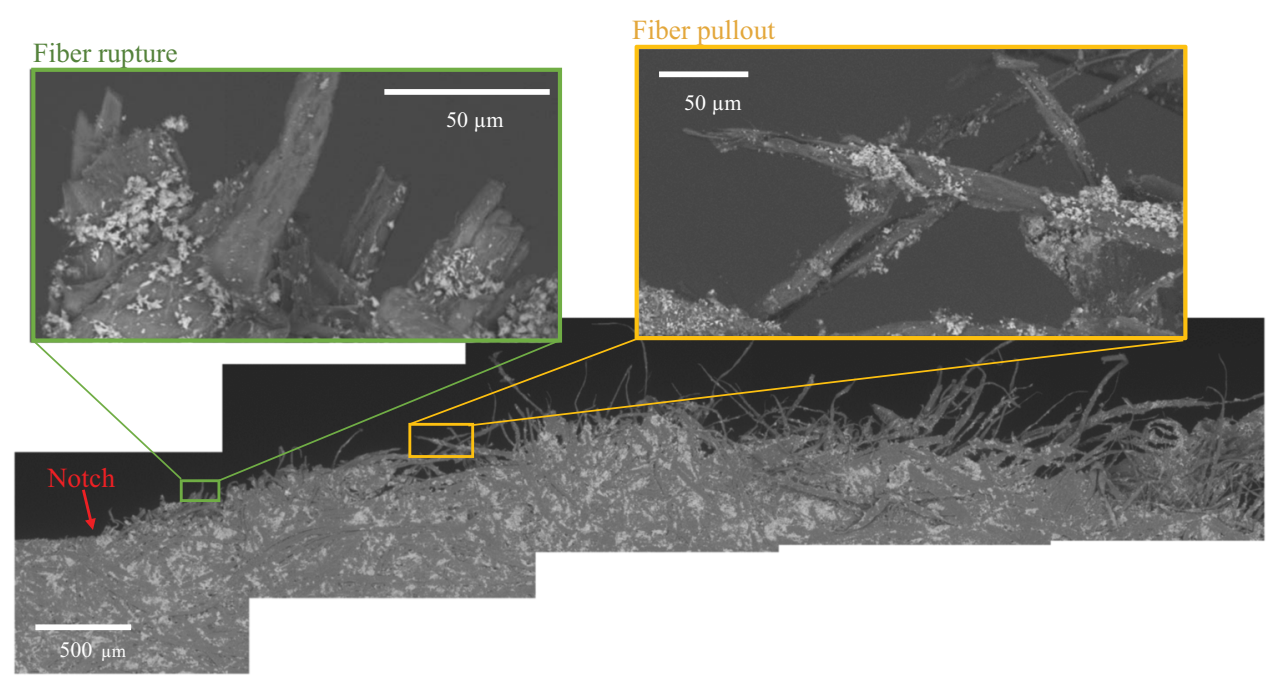

Fig. 4 Intermediate fatigue life crack profiles. Scanning electron microscopy images of a representative, intermediate fatigue life $\left(N_{f}=18501 \mathrm{cycles}\right)$ showed the distinctive fiber rupture and pull-out mechanisms.

Ratchetting and fatigue are the two types of cyclic damage that are observed in materials, and they arise from different damage mechanisms (Fig. 2). When a material is stressed in tension above its yield strength (but not to failure), plastic deformation and damage can accumulate. If the material is then unloaded and then reloaded to a higher stress (and strain), the loading path will be bounded by an envelope defined by its uniaxial tensile behavior. If the loading-unloading hysteresis loop does not close on itself, additional loading cycles will cause progressively larger ratchetting strains to accumulate. Consequently, ratchetting is a static damage mechanism, in which fracture occurs as the tensile fracture strain is reached after a few loading cycles. Ratchetting is observed in a wide range of materials including paper ${ }^{4-9}$, and is most prominent in materials that show significant timedependent (creep) deformation (reviews by Ohno and Kang ${ }^{10,11}$ detail ratchetting and how it is distinguished from fatigue). 


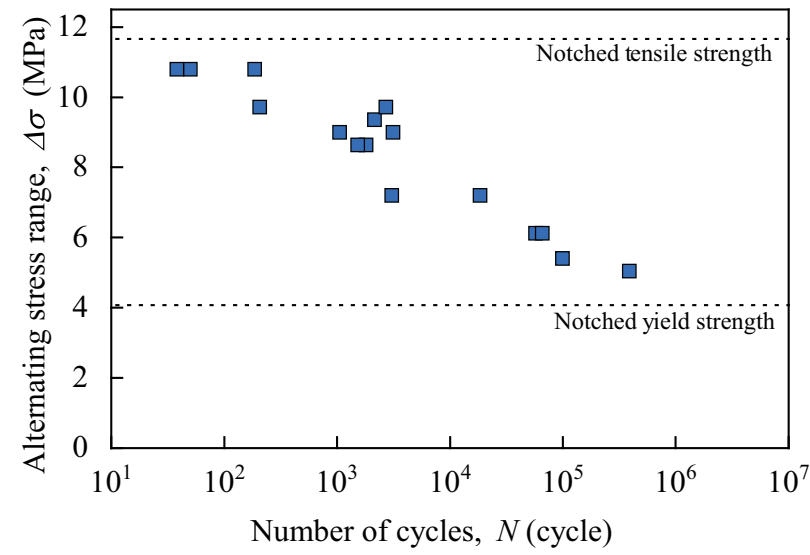

Fig. 5 Stress-Life fatigue behavior of copy paper. The high-cycle fatigue life $\left(N_{f}\right)$ was measured as a function of the applied alternating stress range $(\Delta \sigma)$. The fatigue lives ranged from $<100$ to $\sim 10^{6}$ cycles to failure for stress ranges that were bounded by the notched specimen ultimate and yield strengths, respectively (dashed lines). The error bars were smaller than the symbols used to denote the fatigue lives.

Ratchetting fracture surfaces in paper are hairy and indistinguishable from tensile fracture and creep because the damage mechanisms are essentially the same. However, if the cyclic stress amplitude is reduced so that the specimen lasts for tens of cycles or more, truly cycle-dependent (fatigue) mechanisms can occur.

In this work, specimens were cyclically loaded with peak stresses greater than the yield stress, cyclic damage was accumulated, and the specimens eventually fractured into two pieces within about $10^{6}$ cycles (Fig. 5). The number of cycles to failure, $N_{f}$, as a function of alternating stress range, $\Delta \sigma$, showed the power law trend commonly observed in metallic materials ${ }^{12}$. The first indications of a distinct fatigue damage accumulation mechanism in paper became clear when we viewed crack profiles of the test specimens (Fig. 3). The short life fatigue specimen (Fig. 3b), which lived around 200 cycles at a stress range of 9.72 $\mathrm{MPa}$, had a very fibrous and hairy fracture path like the tensile test specimens (Fig. 3a). However, the fatigue crack profiles of the intermediate and long-life specimens were quite different. The fibers adjacent to the notch of the intermediate life specimen (Figs. 3c and Fig. 4) $\left(N_{f}=18,501\right.$ cycles at a stress range of 7.2 $\mathrm{MPa}$ ) were broken instead of pulled out, so the fracture surface near the precut notch was not hairy. The crack growth mechanism transitioned back to the hairy, fiber pull-out mechanism when the fatigue crack was too long and the fracture toughness of the specimen was exceeded. A longer fatigue damage region (fractured fibers) was observed in the crack profile of long-life specimens (Fig. 3d) $\left(N_{f}=98,808\right.$ cycles at stress range of $\left.5.4 \mathrm{MPa}\right)$. Inorganic fillers in the paper were visible as bright particles in the images (Figs. 3 and 4), and were found throughout the tensile, fatigue, and creep fracture surfaces. Based on the crack profiles of the specimens, we concluded that the high-cycle fatigue damage mechanism in paper was distinct from the mechanism observed in other failure modes.

Crack growth rates characterize cycle-dependent behavior. Static, time-dependent, and cyclic damage accumulate in most organic and inorganic materials in ambient laboratory air environments. These three types of damage accumulation mechanisms enable subcritical crack growth that can cause delayed failures in specimens, components, and structures. Distinguishing pure cyclic fatigue from static and time-dependent crack growth is challenging because all three mechanisms can synergistically and simultaneously cause crack growth during cyclic loading

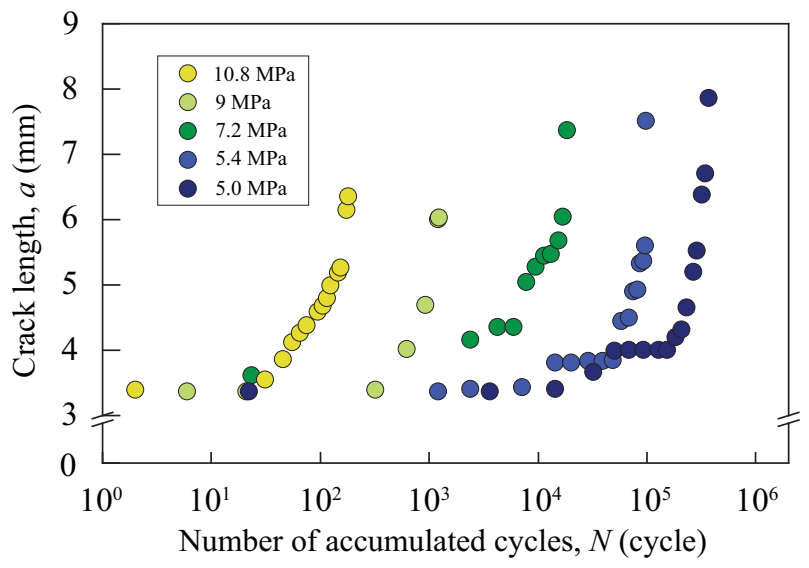

Fig. 6 Crack extension during fatigue loading. Digital image correlation was used to determine the crack length $(a)$ as a function of the number of accumulated cycles $(N)$. The legend indicates the alternating stress range for each specimen $(\Delta \sigma)$. The error bars were smaller than the symbols used to denote the crack lengths.

conditions. An effective strategy for isolating damage mechanisms is to monitor the growth of cracks under strategically selected, controlled-loading and environmental conditions. Static and cyclic loading contributions can, in principle, be separated using smooth (straight gauge) specimens and applying strategies described by Evans \& Fuller ${ }^{13}$. For example, this approach can distinguish between stress corrosion cracking (a static loading, time-dependent, subcritical crack growth mechanism) and cyclic fatigue crack growth in some polycrystalline ceramics ${ }^{14}$. However, analysis of stress-life $(\Delta \sigma-N)$ data is often inconclusive because of the scatter intrinsic to the (total life) experiment (note Fig. 5). Similarly, the standard folding endurance test methods for straight gauge paper specimens superimpose static and cyclic loading modes (TAPPI T423 and T511). The mechanisms that enable fatigue damage accumulation are classified based on how and where damage accumulates as a crack grows ${ }^{12}$. Therefore, we used crack rate growth measurements to isolate and characterize the cyclic damage accumulation in high-cycle fatigue.

In conventional, dense structural materials it is relatively easy to define the location and size of a crack because it is bounded by free surfaces. Unfortunately, it is very difficult to clearly observe and define cracks in the heterogeneous, porous, heavily bridged fiber networks found in wood fiber-based paper and packaging materials. Instead, we developed a digital image correlation-based analysis strategy that quantified the fatigue damage zone extent (size) and growth rates ("Methods"). The spatial distribution of the experimentally measured strains were used to identify continuous, crack-like regions where the fiber network had failed. This is the first application of this type of approach to heterogeneous fiber composites. We then were able to measure the crack growth rates and recorded specimen lives under fatigue $(\Delta \sigma=5.0$ to $10.8 \mathrm{MPa})$, creep $(\sigma=6.8 \mathrm{MPa})$, and alternating creep-fatigue loading conditions.

The fatigue crack growth rates systematically decreased as the applied stress amplitude was reduced (see Fig. 6 and Table 1). The maximum fatigue crack growth rate was $3.37 \times 10^{-2} \mathrm{~mm}$ cycle $^{-1}$ for the specimen with the highest stress amplitude and one of the shortest lives ( $\Delta \sigma=10.8 \mathrm{MPa}, N_{f}=187$ cycles). The maximum growth rate dropped by three orders of magnitude $\left(2.91 \times 10^{-5} \mathrm{~mm} \mathrm{cycle}{ }^{-1}\right)$ when the driving force was reduced $(\Delta \sigma=5.0 \mathrm{MPa})$ and the specimen lasted 2040 times longer (381,628 cycles). Table 1 shows that as the alternating stress range was increased, the average and maximum crack growth rates of paper under cyclic loading increased. The fatigue crack growth 


\section{Table 1 Fatigue crack growth rates in copy paper.}

\begin{tabular}{lll}
$\begin{array}{l}\text { Alternating stress } \\
\text { range, } \boldsymbol{\Delta} \boldsymbol{\sigma}(\mathbf{M P a})\end{array}$ & $\begin{array}{l}\text { Average } \mathbf{~ d a} / \mathbf{d N} \\
\left(\mathbf{m m} \mathbf{c y c l e}^{-\mathbf{1}}\right)\end{array}$ & $\begin{array}{l}\text { Maximum da/dN } \\
\left(\mathbf{m m} \mathbf{c y c l e}^{-\mathbf{1}} \mathbf{)}\right.\end{array}$ \\
\hline 5.0 & $8.31 \times 10^{-6}$ & $2.91 \times 10^{-5}$ \\
5.4 & $3.97 \times 10^{-5}$ & $3.31 \times 10^{-4}$ \\
7.2 & $1.74 \times 10^{-4}$ & $6.92 \times 10^{-3}$ \\
9.0 & $1.23 \times 10^{-3}$ & $1.42 \times 10^{-3}$ \\
10.8 & $1.52 \times 10^{-2}$ & $3.37 \times 10^{-2}$ \\
\hline
\end{tabular}

Digital image correlation was used to experimentally measure the average and maximum fatigue crack growth rates $(\mathrm{da} / \mathrm{dN})$ as a function of the alternating stress range $(\Delta \sigma)$ at a constant load ratio $(R=0.1)$

was not correlated with the linear elastic fracture mechanics parameter, $\Delta K$ (Supplementary Discussion). However, the fatigue crack growth rate was sensitive to the applied stress and loading mode (constant, monotonically increasing, and cyclic), so we examined the creep and fatigue damage accumulation mechanisms and their synergistic interactions.

Creep-fatigue interactions. Like with most materials, it was difficult to tell only from the total life data how creep and fatigue processes interact in paper ${ }^{12}$. Figure 5 shows the wide variation in fatigue life, which is typical of straight gauge specimens. To lend new insight into the interaction of creep and fatigue in paper, we used our digital image correlation strainfield analysis approach to identify the crack sizes and then characterized damage accumulation with fatigue crack growth rates. The interactions between and relative importance of creep and fatigue became clear.

Six specimens were tested with the same max stress of $6.8 \mathrm{MPa}$. Two specimens, F1 and F2, were subjected to fatigue only and lived 57,329 and 65,970 cycles (at $1 \mathrm{~Hz}$ ). Two specimens, $\mathrm{C} 1$ and $\mathrm{C} 2$, underwent creep, but were stopped before completion at 203,630 and 221,400 s due to crack arrest. A final two specimens, CF1 and CF2, were subjected to about 36,000 and $30,000 \mathrm{~s}$ of creep then fatigued to total failure. Figure 7 shows the number of accumulated cycles for these six specimens vs. the crack length determined using digital image correlation and thresholding ("Methods"). The specimens subjected to constant (creep) loading before fatigue, CF1 and CF2, lived much longer than the specimens that were only subjected to cyclic loading $(354,741$ and 192,498 cycles, respectively). The increase in fatigue life by up to 6.2 times was surprising given the general perception that creep damage is more important than fatigue for packaging materials. It was clear that the creep and fatigue damage mechanisms in paper are different, and there is a synergistic interaction between creep and fatigue that can enhance fatigue resistance.

Supplementary Fig. 1 shows the computed fatigue crack growth (FCG) rates for the fatigue only specimens, F1 and F2, and the fatigue portion of the creep-fatigue specimens, CF1 and CF2. Crack growth rates were not measured for creep, as they were much smaller than fatigue and asymptotically approached zero due to crack arrest. It is worth noting that the variability in FCG rates decreased when specimens were first subjected to creep (constant) loading. The crack growth rates reveal that specimens subjected to fatigue only grew at an average rate of $5.25 \times 10^{-5}$ and $5.55 \times 10^{-5} \mathrm{~mm}$ cycle $^{-1}$. Given these specimens had an alternating stress of $6.1 \mathrm{MPa}$, the FCG rates were consistent with the trends in Table 1. However, when the paper was first subjected to constant loading, their crack growth rates decreased nearly an order of magnitude to $5.33 \times 10^{-6}$ and $6.01 \times 10^{-6} \mathrm{~mm} \mathrm{cycle}{ }^{-1}$.

Finally, we looked at the effect of mixed constant (static) and cyclic loading on the fracture surfaces. Figure 8 shows crack profiles for the two creep-fatigue specimens. The specimens show

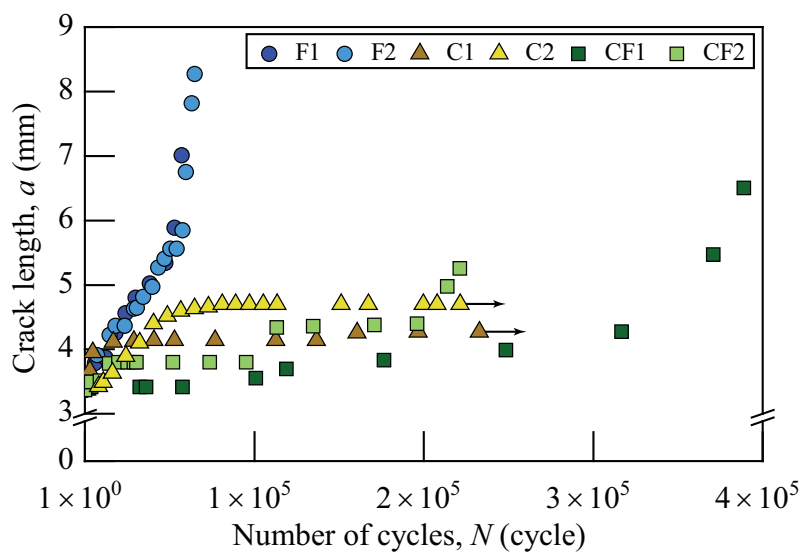

Fig. 7 Crack extension during fatigue, creep, and creep-fatigue loading. The crack length $(a)$ as a function of the number of accumulated cycles $(N)$ during the cyclic and constant stress-loading experiments was measured using digital image correlation. In both creep experiments, C1 and C2, the arrows indicate that the tests were halted before failure due to crack arrest. The error bars were smaller than the symbols used to denote the crack lengths.

a mix of fiber rupture and fiber pullout. This was expected given the hairy fracture surface profile for creep damage and fiber rupture in intermediate and long-life fatigue fracture surface profiles (Fig. 3). However, it was apparent that there was more fiber rupture in the regions undergoing creep or fatigue than in the section of the paper that experienced overload in Fig. 8. Since there was some fiber pullout in the fatigue crack region, this could be evidence that in addition to affecting the fatigue crack growth rate, creep can also impact the local failure mechanism of the fiber matrix.

It is clear from the fracture surfaces and crack growth rate trends that wood fiber pulp-based paper has a distinct high-cycle fatigue damage accumulation mechanism. During tensile fracture, creep, and ratchetting, the fibers deformed and moved relative to one another ${ }^{1,15}$. Ultimately, the interfiber bonds failed because they were weaker than the fibers themselves. In contrast, during high-cycle fatigue the fibers fractured. In principle, the preferred crack path and micromechanism could change with the fiber network topography, composition, specimen geometry, and environment. Paper fiber networks have several characteristic length scales that define the types of locations where high-cycle fatigue can cause fibers to fracture. The fibers in this study ("Methods" and ref. ${ }^{3}$ ) had an average length of $1.4 \mathrm{~mm}$ and diameter of $22 \mu \mathrm{m}$. Both the higher and lower fiber density regions (also known as floc and interfloc, respectively) had characteristic length scales on the order of several millimeters ${ }^{3}$. One could argue that an interfloc fatigue crack path should have been energetically favored because fewer fibers rupture per unit crack extension. However, we did not find evidence of such a preferred fatigue crack path. Thus we concluded that there were two possible types of fiber network locations that could accumulate high-cycle fatigue damage. First, contact damage (fretting) could have occurred where the fibers touched each other. These contacts could occur at interfiber bonds formed when the paper was manufactured, or when fibers moved into contact during deformation. Second, individual fibers could have accumulated high-cycle fatigue damage in the regions between the interfiber bonds. In principle, these two classes of high-cycle fatigue damage accumulation can operate simultaneously. However, the dramatic reductions in fatigue crack growth rate after exposure to creep-loading conditions suggested that high-cycle fatigue damage occurred preferentially at the interfiber bonds 

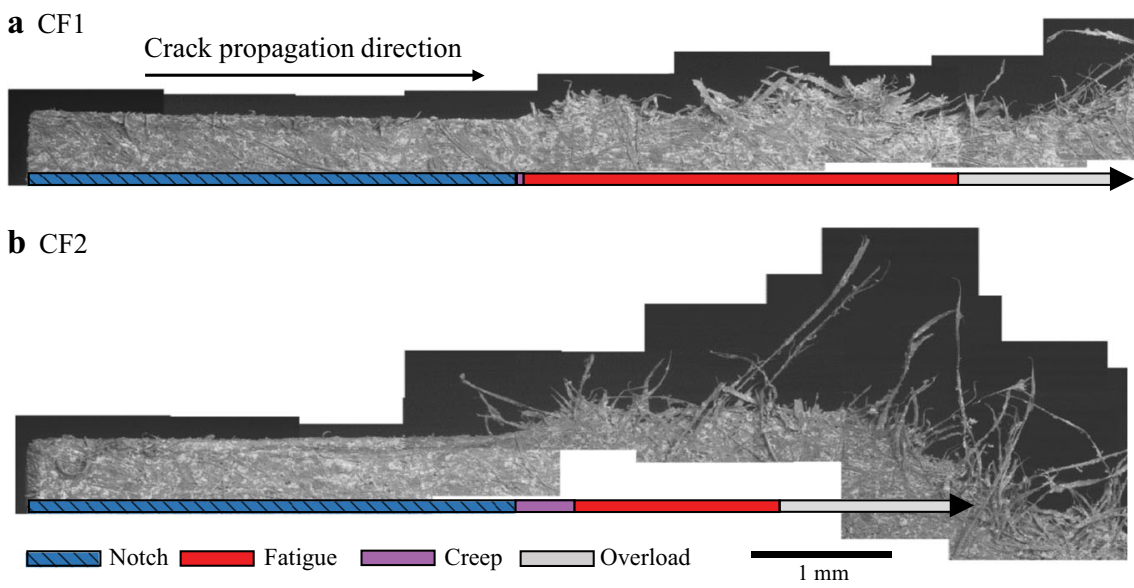

Fig. 8 Creep-fatigue loading crack profiles. Scanning electron microscopy was used to image the specimens (a CF1 and b CF2) subjected to creep followed by fatigue to failure. The cracks grew across the specimen from the cut notch tip as indicated in the figure. The fatigue and creep portions of the fracture surface show less fiber pullout than the overload sections (full specimen width is not shown). Digital image correlation was used to determine the crack length associated with creep, fatigue, and overload.

because creep is dominated by strain accumulation at interfiber bonds ${ }^{15}$. These new insights will allow for the fiber properties and network topography in paper to be engineered for high-cycle fatigue damage resistance.

\section{Methods}

Specimen preparation and mechanical testing. Notched and unnotched specimens were prepared from letter-sized copy papers with grammage of $75 \mathrm{~g} / \mathrm{m}^{2}$ (135885-WH, Staples, Framingham, MA). The specimens were cut into $12.5 \mathrm{~mm}$ wide and $88-\mathrm{mm}$ long strips in the cross-machine direction (CD) using the Sizzix Eclipse 2 paper cutting machine (Sizzix, Lake Forest, CA). For notched specimens, a single notch spanning $27 \%$ of the width was also cut in one edge of the specimens. The actual gauge length of a specimen was $50 \mathrm{~mm}$, since each grip end was $19 \mathrm{~mm}$ long. The specimen dimensions were modified from TAPPI standard, T494. A random speckle pattern was airbrushed (Iwata, Portland, OR) on the specimens for feature tracking purposes in digital image correlation (DIC). As specified by TAPPI standard T402, all specimens were preconditioned at $23{ }^{\circ} \mathrm{C}$ and $35 \% \mathrm{RH}$ for an hour and conditioned at $23{ }^{\circ} \mathrm{C}$ and $50 \% \mathrm{RH}$ for at least four hours prior to testing in a SH-241 (ESPEC Corp., Osaka, Japan) benchtop temperature and humidity chamber. The standard methods detailed in the TAPPI standards are functionally identical to the related ASTM standards D828, D685, and E111.

All mechanical tests were conducted with a $100 \mathrm{~N}( \pm 0.5 \%)$ Instron $2530-427$ static load cell with the Instron 5848 (Instron, Norwood, MA) load frame equipped with a magnetic linear encoder with a $20-\mathrm{nm}$ resolution (Heidenhain, Shaumburg, IL) placed on a benchtop vibration isolated platform (BM-4, Minus K Technology, Inglewood, CA). The specimens were gripped with 2716-016 serrated mini wedge grips from Instron. A small preload $(<3 \mathrm{~N})$ was applied to remove any slack in the specimen and obtain focused images for the tests. Tensile tests were conducted in displacement control mode at a rate of $3 \mathrm{~mm} \mathrm{~min}^{-1}$. Fatigue tests in tension were conducted in load control mode at $1 \mathrm{~Hz}$ with a minimum to maximum load ratio $(R)$ of 0.1 . Creep tests were also conducted in load control mode. LabView 7.1 (National Instruments, Austin, TX) software was used to control the actuator and to collect the tensile data from the Instron. Data analysis was performed in Microsoft Office Excel 2010 and Python 2.7.3. The uniaxial tensile behavior and analysis methods were detailed in ref. ${ }^{3}$. Briefly, 60 specimens were tested for each of the two principal orientations, machine direction (MD), and cross-machine direction (CD). For each specimen the tensile strength, tensile fracture strain, and tensile energy absorption (TEA) were calculated as specified in T494. A constant, nominal thickness of $100 \mu \mathrm{m}$ was determined using scanning electron microscopy images of Spurr's epoxy infiltrated and polished cross-sections of the paper (see ref. ${ }^{3}$ ). The elastic modulus was calculated from the linear portion of a stress-strain curve consistent with the methods detailed in ASTM E111. The linear elastic part of the curve was determined by fitting a linear regression line from the first data point to the data point where the coefficient of determination, $\mathrm{R}^{2}$, was 0.999 . The last data point of the linear portion was recorded as the yield point. The tensile energy absorption, the energy per unit area of the specimen, was defined as the area under load-displacement curve up to the failure point and calculated from the experimental data by numerically integrating using the trapezoidal rule.

A 24 megapixel $(6000 \times 4000)$ Nikon D3300 DSLR camera with a Nikon macro lens was used to capture images of specimens before and during experiments. An Arduino Mega microcontroller with custom firmware was used to trigger the camera and to collect images at a rate of $1 \mathrm{~Hz}$ that were synchronized with the cross-head displacement and load cell data. For fatigue tests, the camera was triggered every $1 \mathrm{~min}$ ( 60 cycles), and at each triggering point a total of nine phaselagged images were taken every $1.125 \mathrm{~s}$ to reconstruct the sine wave cycle. Fracture surfaces and crack profiles were imaged using a Hitachi TM3030 benchtop scanning electron microscope (SEM) in "COMPO" backscatter imaging mode.

The spatial distribution of deformation and strains were evaluated by an in house DIC programs developed in Python ${ }^{3,16}$, which were based on both conventional feature tracking and optical flow (Lucas-Kanade) algorithms ${ }^{17,18}$. Strain maps based on the motion of tracked positions were plotted using a linearly interpolated Voronoi tesselation scheme. The noise level in the tracked displacement and strain values were determined using a rigid body translation calibration scheme. Before each experiment the camera was translated known distances (optical encoder resolution better than $1 \mu \mathrm{m}$ ), and features were tracked using DIC. The measured displacement error was up to 0.1 pixel, which corresponded to a strain noise floor of $10^{-6}$ ). The color bars used to represent the strains were then selected to insure that apparent differences in color were associated with significant differences in strain. The line width resolution of the images was determined with a 1951 USAF microscope resolution target, and was better than $20 \mu \mathrm{m}$.

The individual fibers and the fiber network architecture were characterized using optical microscopy techniques as detailed in $^{3}$. Briefly, a full U.S. standard letter sheet of the paper $(215.9$ by $279.4 \mathrm{~mm}$ ) was torn into 2 by $3 \mathrm{~cm}$ pieces and soaked overnight in cold water. A British disintigrator was used to create a slurry that was passed through a fiber quality analyzer (OpTest Equipment Inc., ON Canada) ${ }^{19,20}$. The polarized light optical system was used to measure the fiber length, width, and geometry (curl and kink). The percentage of fines was determined using a length cutoff of $0.2 \mathrm{~mm}$. After two runs, the average fiber length and diameter were $1.359 \mathrm{~mm}$ and $21.6 \mu \mathrm{m}$, respectively. The fiber total kink angle was $30.2^{\circ}$ with a mean kink length of $0.73 \mathrm{~mm}$. The arithmetic average fines were $15.5 \%$. The characteristic length scales of the floc and interfloc regions were evaluated using a lineal path correlation analysis of visible light transmission through the paper $3,21,22$. The fast Fourier transform (FFT) of the images were low pass filtered to isolate the floc and interfloc regions, and the inverse FFT was applied to the power spectrum of the filtered image data. The images were thresholded, and a lineal path correlation method was used to determine the characteristic length scales ${ }^{23,24}$. The floc and interfloc regions in the copy paper samples used in this study were about $3 \mathrm{~mm}$ and nearly isotropic ${ }^{3}$. The presence of inorganic fillers was noted in the scanning electron micrographs of fractured specimens, but their chemistry and mass fraction were not determined.

Crack length and growth rate measurements. In the case of our paper specimens, it was impossible to use transmitted and/or reflected visible light images to directly define the length of the crack during our experiments because crack lengths were underestimated in the optical images. As shown in Fig. 9, the porous fiber network structure and diffuse (extrinsic bridging) damage made it difficult to distinguish the crack. The experimentally measured strain fields were used to identify the extent of the crack using a thresholding strategy. A threshold strain of 0.05 , which is 1.5 times greater than the failure strain of unnotched CD specimens in uniaxial tension (Supplementary Table 1), was used to define material that had failed (or at least been significantly damaged). The extent of the damage, and the location of the crack tip, was determined from the continuous region with strains above the threshold level (along the midline of the original cut notch location). It is important to note that the crack sizes found using the digital image correlation were 


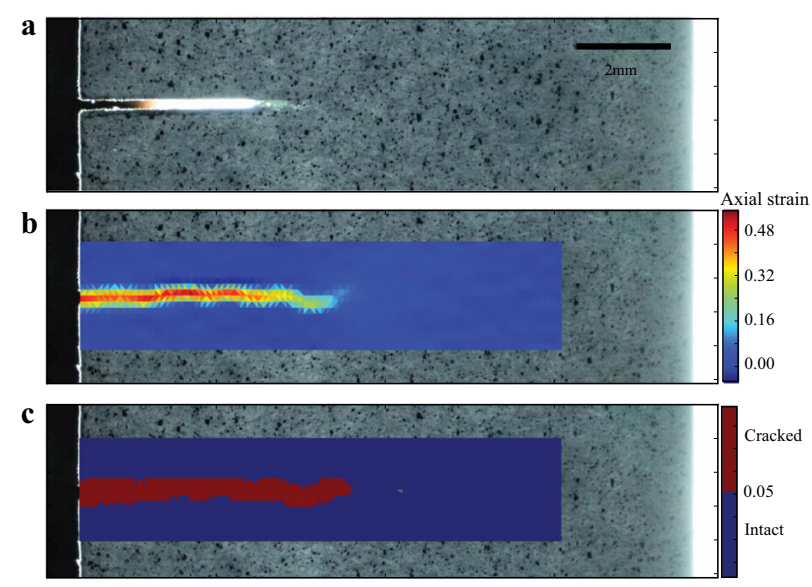

Fig. 9 Determining crack length with image analysis. Optical images and thresholded digital image correlation strain map overlaps showing extent of crack growth. It was difficult to discern the crack tip in the a transmitted light optical images, but the strain maps $(\mathbf{b}, \mathbf{c})$ allowed for measurement of the crack length.

much larger than the apparent crack sizes in the optical images because changes in light transmission only become visible once all the fibers have moved from their initial positions, which occurs long after the fiber or interfiber failure. The details of these methods are important to consider when comparing our results to previous studies of crack growth in paper ${ }^{25-37}$.

A series of threshold levels were evaluated and the influence on crack growth rate was determined: changes in the threshold level caused minor shifts in crack size, but the influence on growth rates was small. Supplementary Fig. 2 shows the effect of the selected axial thresholding strain on the normalized apparent crack length $(a / w)$ for two fatigue specimens at a particular cycle in the test. To best represent crack growth, the selected thresholding strain should strain should be above the tensile fracture strain, 0.035 , and ideally above the strain plus the standard deviation, 0.039 . This criteria guarantees that the material had separated in the selected strain of 0.05 . The plot shows that between 0.04 and 0.06 thresholding strains, there is a linear (stable) relationship with respect to crack length. Eventually the thresholding strain becomes so high that the apparent crack length is the normalized length of the notch, 0.27 . Furthermore, Supplementary Table 2 shows the varying the thresholding strain between $0.04,0.05$, and 0.06 results in minimal change to the fatigue crack growth rates.

Crack length versus number of accumulated cycles ( $a$ vs. $N$ ) curves for all the fatigue and creep tests shown in this paper (Figs. 6 and 7) were obtained using this unique crack length measurement method. Supplementary Fig. 3 shows a representative $a$ vs. $N$ plot of a specimen cyclically loaded with an alternating stress range of $6.12 \mathrm{MPa}$. Per ASTM standard E-647, the crack growth rate, da/dN, was calculated from the slope of a linear regression of five consecutive data points (highlighted in the figure), and the crack growth rate was attributed to the mid (third) data point of the group. The analysis window was then advanced by one data point, and the analysis was repeated.

\section{Data availability}

The data sets generated during and/or analyzed during this study are available for download from the Georgia Institute of Technology SMARTech Repository at smartech. gatech.edu (http://hdl.handle.net/1853/62477).

Received: 5 September 2019; Accepted: 26 March 2020;

Published online: 15 May 2020

\section{References}

1. Niskanen, K. Mechanics of Paper Products (De Gruyter, Berlin, 2012)

2. Picu, R. C. Mechanics of random fiber networks-a review. Soft Matter 7, 6768-6785 (2011).

3. Na, Y. J. \& Muhlstein, C. L. Relating nonuniform deformations to fracture in uniaxially loaded non-woven fiber networks. Exp. Mechanics 59, 1127-1144 (2019).

4. Coffin, D. W. The creep response of paper. in Advances in Paper Science and Technology: Transactions of the 13th Fundamental Research Symposium. (ed. IAnson, S. J.) 651-747 (2005).
5. Coffin, D. W. Use of the efficiency factor to account for previous straining on the tensile behavior of paper. Nord. Pulp Pap. Res. J. 27, 305-312 (2012).

6. Miksic, A. et al. Deformation, acoustic emission and ultrasound velocity during fatigue tests on paper. in ICEM 14: 14th International Conference on Experimental Mechanics, (ed. Bremand, F.) Vol 6, 42016-1-6 (ICEM, 2010).

7. Miksic, A., Koivisto, J. \& Alava, M. Statistical properties of low cycle fatigue in paper. J. Stat. Mech.-Theory Exp.Number 5, May 2011, P05002-1-25 (2011).

8. Miksic, A., Koivisto, J., Rosti, J. \& Alava, M. Strain fluctuations from dic technique applied on paper under fatigue or creep. 11th International Conference on the Mechanical Behavior of Materials (ICM11) 10 (2011).

9. Viitanen, L., Ovaska, M., Ram, S. K., Alava, M. J. \& Karppinen, P. Predicting creep failure from cracks in a heterogeneous material using acoustic emission and speckle imaging. Phys. Rev. Appl. 11, 024014 (2019).

10. Ohno, N. Recent topics in constitutive modeling of cyclic plasticity and viscoplasticity. Appl. Mechan. Rev. 43, 283-295 (1990).

11. Kang, G. Ratchetting: recent progresses in phenomenon observation, constitutive modeling and application. Int. J. Fatigue 30, 1448-1472 (2008).

12. Suresh, S. Fatigue of Materials. 2nd edn (Cambridge University Press, Cambridge, 2003).

13. Evans, A. G. \& Fuller, E. R. Crack propagation in ceramic materials under cyclic loading conditions. Metall. Transact. A (Phys. Metall. Mater. Sci.) 5, 27-33 (1974).

14. Munz, D. \& Fett, T. Ceramics: Mechanical Properties, Failure Behaviour, Materials Selection (Springer, Berlin; New York, 1999).

15. Haslach, H. W. The moisture and rate-dependent mechanical properties of paper: a review. Mechan. Time-Dependent Mater. 4, 169-210 (2000).

16. Collins, J. G., Dillon, G. P., Strauch, E. C., Lanning, W. R. \& Muhlstein, C. L. Correlating bonded joint deformation with failure using a free surface strain field mining methodology. Fatigue Fract. Eng. Mater. Struct. 39, 1124-1137 (2016).

17. Sutton, M. A., Cheng, M. Q., Peters, W. H., Chao, Y. J. \& Mcneill, S. R. Application of an optimized digital correlation method to planar deformation analysis. Image Vision Comput. 4, 143-150 (1986).

18. Lucas, B. D. \& Kanade, T. An iterative image registration technique with an application to stereo vision (IJCAI). in Proceedings of the 7th International Joint Conference on Artificial Intelligence (IJCAI '81), 674-679 (Morgan Kaufmann Publishers Inc., San Francisco, CA 1981).

19. Meyers, J. \& Nanko. Effects of fines on the fiber length and coarseness values measured by the fiber quality analyzer (fqa). in TAPPI Spring 2005 Technical Conference, 1-8 (TAPPI, Peachtree Corners, GA, 2005).

20. Li, B., Bandekar, R., Zha, Q., Alsaggaf, A. \& Ni, Y. Fiber quality analysis: optest fiber quality analyzer versus $1 \&$ w fiber tester. Ind. Eng. Chem. Res. 50, 12572-12578 (2011).

21. Hladnik, A., Muck, T., Stanic, M. \& Cernic, M. Fast fourier transform in papermaking and printing: two application examples. Acta Polytech. Hung. 9, 155-166 (2012)

22. Bernie, J. P. \& Douglas, W. J. M. Local grammage distribution and formation of paper by light transmission image analysis. TAPPI J. 79, 193-202 (1996).

23. Singh, H., Gokhale, A. M., Lieberman, S. I. \& Tamirisakandala, S. Image based computations of lineal path probability distributions for microstructure representation. Mat. Sci. Eng. A-Struct. 474, 104-111 (2008).

24. Tewari, A., Gokhale, A. M., Spowart, J. E. \& Miracle, D. B. Quantitative characterization of spatial clustering in three-dimensional microstructures using two-point correlation functions. Acta Mater. 52, 307-319 (2004).

25. Makela, P. Engineering fracture mechanics analysis of paper materials. Nord. Pulp Pap. Res. J. 27, 361-369 (2012).

26. Makela, P. \& Fellers, C. An analytic procedure for determination of fracture toughness of paper materials. Nord. Pulp Pap. Res. J. 27, 352-360 (2012).

27. Seth, R. S. \& Page, D. H. Fracture resistance of paper. J. Mater. Sci. 9 1745-1753 (1974).

28. Seth, R. S., Robertson, A. G., Mai, Y. W. \& Hoffmann, J. D. Plane-stress fracture-toughness of paper. TAPPI J. 76, 109-116 (1993).

29. Seth, R. S. Measurement of inplane fracture-toughness of paper. TAPPI J. 78, 177-183 (1995).

30. Wanigaratne, D. M. S., Batchelor, W. J., Conn, A. B. \& Parker, I. H. Image analysis of plastic deformation in the fracture of paper. Appita J. 53, 471-475 (2000).

31. Wellmar, P., Fellers, C., Nilsson, F. \& Delhage, L. Crack-tip characterization in paper. J. Pulp Pap. Sci. 23, J269-J276 (1997).

32. Wellmar, P., Gregersen, O. W. \& Fellers, C. Prediction of crack growth initiation in paper structures using a j-integral criterion. Nord. Pulp Pap. Res. J. 15, 4-11 (2000).

33. Yamauchi, T. \& Hirano, H. Examination of the onset of stable crack growth under fracture toughness testing of paper. J. Wood Sci. 46, 79-84 (2000).

34. Yuhara, T. \& Kortschot, M. T. A simplified determination of the j-integral for paper. J. Mater. Sci. 28, 3571-3580 (1993). 
35. Zechner, J., Janko, M. \& Kolednik, O. Determining the fracture resistance of thin sheet fiber composites-paper as a model material. Compos. Sci. Technol. 74, 43-51 (2013)

36. Coffin, D. W. A fracture-based description for the development of tensile and tear strength in paper. J-FOR-J. Sci. Technol. Forest Products and Processes 6, 29-38 (2018).

37. Lanning, W. R., Javaid, S. S. \& Muhlstein, C. L. Reconciling fracture toughness parameter contradictions in thin ductile metal sheets. Fatigue Fract. Eng. Mater. Struct. 40, 1809-1824 (2017).

\section{Acknowledgements}

The authors gratefully acknowledge the support of the Renewable Bioproducts Institute at the Georgia Institute of Technology. The sponsor had no direct role in the design, collection, analysis, interpretation of data, or production of this article. The authors thank Dr. M. Misra for editorial guidance and Dr. R. Yang for performing the fiber quality analysis.

\section{Author contributions}

Y.N. designed and conducted experiments, collected the data, developed the thresholding crack length measurement scheme, and analyzed the results. S.P. conducted additional experiments, collected the data, and developed the thresholding sensitivity analysis for the method, and analyzed the additional experiments. Y.N. prepared the original version of the manuscript and contributed to the revised paper. S.P. prepared the revised version of the paper. C.M. supervised the study, including preparation of the paper. All authors contributed to finalizing the paper for publication.

\section{Competing interests}

The authors declare no competing interests.

\section{Additional information}

Supplementary information is available for this paper at https://doi.org/10.1038/s43246 020-0025-2.

Correspondence and requests for materials should be addressed to C.L.M.

Reprints and permission information is available at http://www.nature.com/reprints

Publisher's note Springer Nature remains neutral with regard to jurisdictional claims in published maps and institutional affiliations.

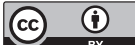

Open Access This article is licensed under a Creative Commons Attribution 4.0 International License, which permits use, sharing, adaptation, distribution and reproduction in any medium or format, as long as you give appropriate credit to the original author(s) and the source, provide a link to the Creative Commons license, and indicate if changes were made. The images or other third party material in this article are included in the article's Creative Commons license, unless indicated otherwise in a credit line to the material. If material is not included in the article's Creative Commons license and your intended use is not permitted by statutory regulation or exceeds the permitted use, you will need to obtain permission directly from the copyright holder. To view a copy of this license, visit http://creativecommons.org/ licenses/by/4.0/.

(C) The Author(s) 2020 